\title{
Fairness Aware Group Proportional Frequency Domain Resource Allocation in L-SC-FDMA Based Uplink ${ }^{*}$
}

\author{
Irfan Ahmed $^{1}$, Amr Mohamed ${ }^{2}$ \\ ${ }^{1}$ College of Computers and Information Technology, Taif University, Taif, KSA \\ ${ }^{2}$ College of Computer Science and Engineering, Qatar University, Doha, Qatar \\ E-mail:i.ahmed@tu.edu.sa \\ Received May 31, 2011; revised July 2, 2011; accepted July 11, 2011
}

\begin{abstract}
This paper presents virtual clusters based proportional fairness and resource allocation scheme for Localized Single Carrier Frequency Division Multiple Access (L-SC-FDMA). L-SC-FDMA has been selected as the uplink transmission scheme in 3GPP Long Term Evolution (LTE) due to its low Peak to Average Power Ratio (PAPR) over OFDMA in general and high rate-sum capacity over Interleaved SC-FDMA in particular. Virtual cluster-based proportional fairness (VCPF) scheduler exploits the link adaptation information available at MAC layer to form virtual clusters. The distributed proportional fairness scheduler ensures a minimum throughput for all users in the coverage area by assigning contiguous RBs, proportional to the throughput and the number of users in a particular cluster or group. Simulations have been performed using practical scenario of uniformly distributed users in Rayleigh faded coverage area and design formulas have been devised for network planning to get the best possible fairness with promising level of quality of service (QoS).
\end{abstract}

Keywords: Radio Resource Allocation, Fairness, LTE

\section{Introduction}

Nowadays, growing demands of broadband services are provoking the deployment of $4 \mathrm{G}$ technologies like long term evolution (LTE) and LTE-Advanced [1]. LTE has paved the way for the prevalent adoption of orthogonal frequency division multiple access (OFDMA) as the key technology for providing high spectrum efficiency and protection against multipath fading. LTE-Advanced technology is expected to provide peak data rates in the order of 1 Gbps in downlink and $500 \mathrm{Mbps}$ in uplink with a 20 $\mathrm{MHz}$ spectrum allocation [2]. OFDM has been selected as transmission technology of most of the other wireless broadband standards. In multiuser application, OFDM can be used as OFDM-TDMA (time division multiplexing access), OFDM-CDMA (code division multiplexing access) or OFDM-FDMA (frequency division multiplexing access). In case of OFDM-TDMA or OFDMCDMA each user has to transmit its signal over the entire spectrum that leads to an averaged-down effect in the presence of deep fading and narrowband interference,

*This work is supported by the Qatar Telecom (Qtel) Grant No. QUEX-Qtel-09/10-10. whereas, in OFDM-FDMA, commonly called OFDMA (orthogonal frequency division multiplexing access) total bandwidth is divided into frequency blocks (one or group of subcarriers).

An OFDMA system is defined as one in which each user occupies a subset of frequency blocks and each block is assigned exclusively to one user at any time, thus the radio resource are allocated in both the frequency (subcarrier) and time domains.

However, despite of numerous advantages of OFDM and OFDMA, their major disadvantage is that their waveforms have high peak to average power ratio (PAPR). High PAPR is problematic for uplink transmission where the mobile transmission power is usually limited. For PAPR reduction, 3GPP-LTE agreed on using single carrier frequency division multiple access (SCFDMA) transmission with cyclic prefix in the uplink. Therefore, In LTE system, OFDMA has been selected as the downlink access scheme and single carrier frequency division multiple access (SC-FDMA) has been chosen as the uplink access scheme [3].

This paper investigates proportional groups based channel dependent resource allocation scheme that pro- 
vides a minimum required throughput with fairness among active users in SC-FDMA LTE uplink, regardless of their distance from the enhanced NodeB (eNB) or the base station. Since SC-FDMA is based on OFDMA with an additional Discrete Fourier Transform (DFT) block in the transmitter and Inverse DFT (IDFT) block in the receiver, the same benefits in terms of multipath mitigation and channel equalization are achievable in addition to low peak-to-average power ratio (PAPR). This property is very important in uplink as the user equipment (UE) is generally a handheld device with limited transmit power capacity.

SC-FDMA has two types of subcarrier mapping: localized SC-FDMA (L-SC-FDMA) and interleaved SCFDMA (I-SC-FDMA). In L-SC-FDMA subcarriers are mapped in contiguous fashion to obtain the frequency selective diversity whereas in I-SC-FDMA, users are assigned distributed subcarriers over the entire bandwidth which provides multi-user diversity. L-SC-FDMA has been chosen for LTE uplink due to high rate-sum capacity [4] and $2.7 \mathrm{~dB}$ (16QAM, 10\% bandwidth usage) lower PAPR [5] as compared to I-SC-FDMA. In literature, the following three types of subcarrier/resource blocks allocation schemes are widely discussed [6]: 1) throughput optimization; 2) proportional fairness; and 3) max-min fairness. Throughput optimization implies that the greater the throughput of subcarrier $j$ for user $i$, the greater the tendency we perform the corresponding subcarrier allocation, proportional fairness schemes states that in order to achieve the proportional fairness the utility function should be $U_{i}\left(f_{i}\right)=\ln \left(f_{i}\right)$, and max-min tends to allocate a subcarrier to a user who has minimum throughput in order to minimize the gap between the minimum and maximum achieved throughput for any user in the cell.

Considerable research work has already been done in scheduling and radio resource allocation for LTE downlink. However, the optimality solutions and scheduling algorithms derived for downlink case, cannot be directly applied to the uplink due to differences in terms of transmission technology (L-SC-FDMA) and network resource constraints (contiguous physical resource blocks (PRB)). For SC-FDMA, a channel scheduling algorithm regardless of the contiguity in frequency resource allocation has been presented in [4]. F. D. Calabrese et al. [7] proposed a search-tree based channel aware packet scheduling algorithm in which resources are equally shared among users.

Proportional fair scheduling for high throughput 3G/ $4 \mathrm{G}$ networks has been investigated in [8,9]. The joint scheduling of OFDM subcarriers with power allocation for uplink of a single cell has been discussed in [9]. The problem has been formulated as a constrained non-linear optimization, then integer subcarrier assignment has been obtained through sub-optimal heuristic to break the ties of allocating partial subcarriers to multiple users. Utility-based proportional fair scheduling for uplink single cell system has been investigated in [8]. A Game theoretical formulation of optimizing the allocation of OFDMA subcarriers and TDMA resource blocks for LTE networks has been presented, which leads to the design of an algorithm that achieves proportional fairness while providing throughput maximization, but this paper does not cater the contingency of subcarriers.

None of the previous work mentioned above considered the real world scenario faced by cellular operators where some users continuously starving from bandwidth and some users (with favorable channel conditions and proximity with eNB) enjoy high throughput. In a uniformly populated coverage area of eNB, the number of distant UEs with low signal-to-noise ratio (SNR) in outer circular ring are larger than the UEs with better SNR and high throughput in inner ring (area closed to eNB).

\section{Contribution}

The main contributions and significance of our work are two-fold:

1) Virtual group based proportional fairness using MAC layer Modulation and Coding Scheme (MCS) information;

2) Frequency domain resource allocation based on virtual groups.

This paper is organized as follows: In Section 2 we describe the problem statement and the way we formulate the problem. In Section 3, a novel MCS based virtual proportional group fairness for frequency domain resource allocation in L-SC-FDMA has been presented. Performance evaluation and comparisons of proposed scheme are provided in Section 4, followed by conclusions in Section 5.

\section{Problem Statement}

We consider the problem of Frequency domain schedul-

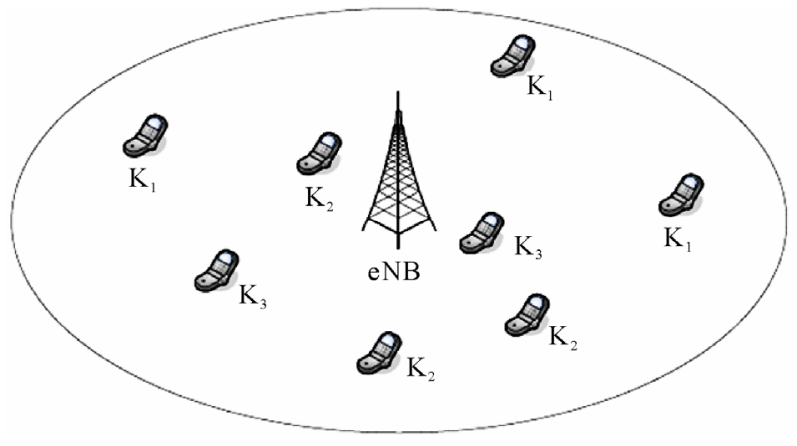

Figure 1. System model. 
ing and resource allocation for the uplink of SC-FDMA in a single sector with the assumption that some other mechanisms are used on top of resource allocation for inter-sector/cell interference mitigation. Fair resource allocation among the users so that they get more or less same quality of service regardless of their position in eNB coverage area has been an important part of Radio Frequency (RF) planning. We present a simple frequency-time domain resource allocation scheme for LTE uplink by subdividing the total users into proportional groups and allocating the resource using proportional marginal fairness scheme. A typical distribution of users in a cell is shown in Figure 1.

\subsection{Problem Formulation}

Let $\mathcal{K}=\{1, \cdots, K\}$ be the set of users in a cell and each user $k \in \mathcal{K}$ has a total transmission power constraint $P_{\text {max }, k}$. The transmission bandwidth $B$ is divided into $N$ physical resource blocks (PRB) indexed by the set $\mathcal{N}=1, \cdots, N$. 3GPP recommends localized SC-FDMA for LTE uplink transmission in which 12 orthogonal consecutive subcarriers constitutes one PRB. We define the Resource Chunk (RC) which consists of a set of consecutive PRBs. Number of PRBs per RC can be computed by dividing total available PRBs by the number of users to transmit, so the minimum size of an RC is equal to one PRB when the number of users is equal to the number of PRB $(N=K)$, which results in maximum number of RCs (equal to number of PRBs). If the number of users exceeds the number of PRBs $(K>N)$, the scheduler randomly selects the users for each epoch until all PRBs are consumed. When PRBs are greater than the number of active users $(N>K)$, i.e., $[q, r]=N / K$ results in quotient $q$ (number of PRBs per RC) and reminder $r$. The remaining $r$ PRBs are evenly assigned to first $r$ RCs.

Let $x_{n, k}$ denote the fraction of RBs allocated to user $k$ such that if RB $n$ is allocated to user $k x_{n, k}$ is equal to 1 . Otherwise, it is equal to 0 . As each RC is allocated exclusively to only one user, we have

$$
\sum_{k=1}^{K} x_{n, k} \leq 1, n=1,2, \cdots, N
$$

Each user is assumed to transmit at the maximum power $P_{k}$ and the power is assumed to be divided equally among all the subcarriers allocated to that user. Equal power allocation is justified by [4] as a fact that the achieved gains are negligible compared to the increase in complexity when optimal power allocation is performed. The maximum transmission power of mobile users is limited as compared to the downlink transmission where the BS has more power and where the varia- tion in user distances from the BS allows the BS to achieve gains by optimizing the power allocation. In order to ensure that all users in a coverage area are able to get some minimum level of throughput $R_{\min , k}$ we exploit the adaptive modulation information at eNB for uplink. Figure 2 depicts the received SNR thresholds on which eNB allocates data rates with QPSK, 16QAM, and 64QAM modulation schemes to users for uplink transmission. Based on this information we divide the total users in three groups $K_{1}, K_{2}$, and $K_{3}$ supporting the modulation schemes QPSK, 16QAM, and 64QAM, respectively, as shown in Figure 3. Then, scheduling algorithm divides the resources in such a way that each user gets guaranteed minimum level of QoS (i.e., throughput in our case).

\subsection{Inequality Constraint Optimization Problem}

We have to solve the following optimization problem to maximize the sum of user utilities $U$ as

$$
\max \sum_{i=1}^{v} \sum_{k=1}^{K_{i}} U_{k}\left(\mathcal{I}_{R C, k}, P_{k}^{R C}\right)
$$

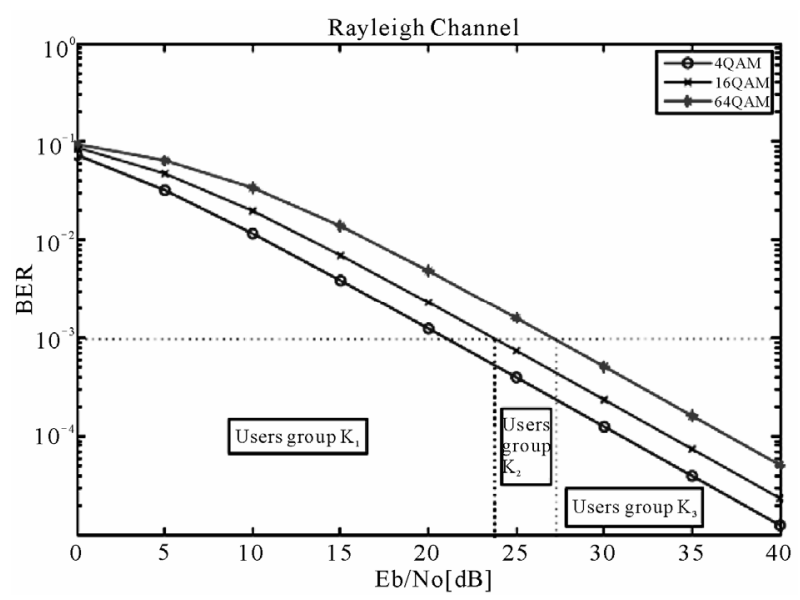

Figure 2. Static link adaptation based users grouping.

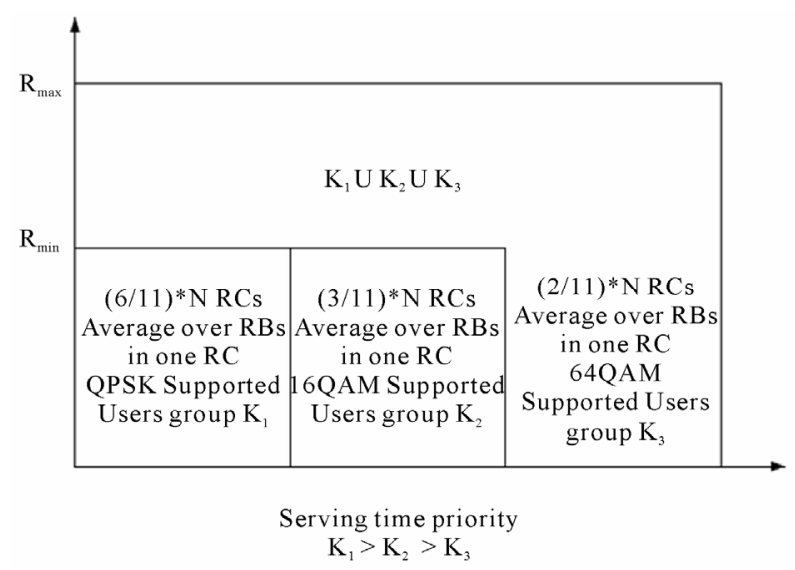

Figure 3. Group priority. 
subject to

$$
\begin{gathered}
P_{k} \leq P_{k, \text { max }} ; \forall k=1, \cdots, K_{i} \\
\sum_{k=1}^{K} x_{n, k}=1 ; \forall n=1, \cdots, N_{K_{i}}
\end{gathered}
$$

where $v$ is the number of user groups, $i=1, \cdots, v$, $\mathcal{I}_{R C, k}$ is the number of radio resource blocks allocated to the user $k$, and $P_{k}^{R C}$ is the average power allocated by the user $k$ to assigned resource block. In general, the utility function is a set of services that determine the user satisfactions in terms of throughput, delay or other QoS criteria. In our case, one example that considers merely throughput maximization is to define the utility function as the effective throughput that a user can achieve with the given radio resource blocks and received SNR $\eta_{n, k}$ as follows:

$$
R\left(\mathcal{I}_{\mathrm{RC}, k}, P_{k}^{R C}\right)=\sum_{n=1}^{N} x_{n, k} \frac{B}{N} \log _{2}\left(1+\frac{\eta_{n, k}}{\Gamma}\right)
$$

where $\Gamma$ is called SNR gap [11]. It indicates the difference between the SNR needed to achieve a certain transmission rate for practical M-QAM and the theoretical limit (Shannon capacity)

$$
\Gamma=-\frac{2}{3} \ln (5 \mathrm{BER})
$$

In SC-FDMA, the noise contribution of highly attenuated subcarriers can be rather large, therefore Minimum Mean Square Error (MMSE) equalizer is preferred over Zero Forcing (ZF) equalizer [10]. The SNR at the output of MMSE equalizer, when each user is assumed to transmit at maximum power $P_{k}=P_{k \text { max }}$ and the power is to be divided equally among all the RCs allocated to that user, is given by [4]

$$
\eta\left(P_{k}, N_{\mathrm{RC}, k}\right)=\left[\frac{1}{\frac{1}{N_{\mathrm{RC}, k}} \sum_{i \in I_{s u b, k}} \frac{\eta_{i, k}}{\eta_{i, k}+1}}-1\right]^{-1}
$$

where $\eta_{i, k}=P_{k} H_{i, k} / \sigma_{n}^{2}$, and $\sigma_{n}^{2}$ is the noise power density.

$H_{i, k}$ is the channel gain when subcarrier $i$ is allocated to user $k, I_{s u b, k}$ is the set of subcarriers assigned to user $k$. Practically, the channel gain depends upon various factors, including thermal noise at receiver, receiver noise figure, antenna gains, distance between transmitter and receiver, path loss exponent, log normal shadowing and fading, hence we can write

$$
H_{i, k}=-\varrho-p 10 \log _{10} d_{k}-\zeta_{i, k}+10 \log _{10} F_{i, k}
$$

In above equation, $\varrho(83.46 \mathrm{~dB})$ is a constant de- pending upon thermal noise at receiver, receiver noise figure, and antenna gains, $p$ (3.5) is path loss exponent, $d_{k}$ is the distance in $\mathrm{Km}$ from UE $k$ to eNB, $\zeta_{i, k}$ (10.5 dB) is shadowing parameter modeled by a normally distributed random variable with standard deviation $8 \mathrm{~dB}$, and $F_{i, k}$ corresponds to Rayleigh fading.

\section{Group Fairness Scheduling Algorithm}

We divide the number of active users at each epoch (i.e., transmission time interval or TTI) into $v$ number of groups, which is equal to number of supported modulation schemes in transmission technology as,

$$
K=\left\{K_{1}, K_{2}, K_{3}, \cdots, K_{v}\right\}
$$

The users in each group contend for RBs separately. The maximum number of RBs that can be allocated to $K_{1}$ group is given by

$$
N_{K_{1}}=\frac{\log _{2}\left(M_{\max }\right) / \log _{2}\left(M_{K_{1}}\right)}{\log _{2} \prod_{i=1}^{v}\left(M_{\max }-M_{K_{i}}\right)} \frac{N^{2} \cdot K_{1}}{\sum_{i=1}^{v} \Psi_{i} K_{i}}
$$

where

$\Psi_{i}=N \log _{2}\left(M_{\max }\right) /\left(\log _{2}\left(M_{K_{i}}\right) \log _{2} \prod_{j}^{v}\left(M_{\max }-M_{K_{j}}\right)\right)$

$M_{\max }$ is the largest constellation size supported by $M$-ary signaling and $M_{K_{1}}$ is the constellation size supported by users in group $K_{1}$. Similarly, $N_{K_{2}}, N_{K_{3}}$, and so on.

Static link adaptation technique has been used to group the users. In this method, the instantaneous SNR of each user is compared with the theoretical BER-SNR curves in fading channel as shown in Figure 3, and based on the received SNR (modulation scheme) each user has been allocated a specific group. For Rayleigh fading channel the SNR corresponding to target BER $\left(P_{b}^{\text {target }}\right.$ ) for rectangular MQAM signaling can be computed as [11, (6.61)]

$$
\mathrm{SNR}=\frac{2\left(1-\frac{2 P_{b}^{\text {target }}}{\hat{\alpha}_{M}}\right)^{2}}{\left[1-\left(1-\frac{2 P_{b}^{\text {target }}}{\hat{\alpha}_{M}}\right)^{2}\right] \hat{\beta}_{M}}
$$

where $\hat{\alpha}_{M}=2(\sqrt{M}-1) / \sqrt{M} \log _{2} M$ and $\hat{\beta}_{M}=3 \log _{2} M /(M-1)$.

In Figure $3 R_{\min }$ is the minimum average throughput that will be guaranteed with $N_{\mathrm{SC}}^{\mathrm{RB}}$ number of subcarriers per RB and $N_{\text {symbol }}$ number of OFDM symbols per TTI, and is given by: 


$$
R_{\text {min }, i}=\frac{N_{\mathrm{SC}}^{\mathrm{RB}} N_{\text {symbol }}}{\operatorname{TTI}(\mathrm{msec})} \frac{N_{K_{i}}}{K_{i}}, \quad i=1, \cdots, v
$$

and

$$
R_{\min }=\inf _{i \in(1, \cdots, v)} R_{\min , i}
$$

Since the optimization problem in (2) has a nonlinear objective function with nonlinear and discrete constraints that requires a complex combinatorial comparison, we present a resource chunk allocation algorithm that maximizes the sum of user marginal utility function in user group.

Marginal utility is defined as the difference between the utility obtained when chunk $n$ is allocated to user $k$ and the utility of user $k$ in the absence of a chunk allocation at each epoch [8]

$$
\Lambda_{n, k}=U\left(\ln \left(R_{k} \mid\{n\} \in N_{\mathrm{RC}, k}\right)\right)-U\left(\ln \left(R_{k} \mid\{n\} \notin N_{\mathrm{RC}, k}\right)\right)
$$

\subsection{Algorithm}

This algorithm applies independently and identically on each group.

Initialization:

Set of potential candidate users,

$$
\mathcal{C}^{t}=\left\{I_{\mathrm{can}, 1}, I_{\mathrm{can}, 2}, \cdots, I_{\mathrm{can}, C}\right\}
$$

Set of $K$ number of randomly selected users from $C$ number of candidates,

$$
\mathcal{K}^{t}=\left\{I_{\text {user }, 1}, I_{\text {user }, 2}, \cdots, I_{\text {user }, K}\right\} \subseteq \mathcal{C}^{t}
$$

Set of resource chunks RCs,

$$
\mathcal{L}^{t}=\left\{I_{\mathrm{RC}, 1}, I_{\mathrm{RC}, 2}, \cdots, I_{\mathrm{RC}, L}\right\}
$$

where $L=$ total number of RBs in B)/(Number of RBs per user).

Objective: To assign an RC $n$ to user $k$ in order to maximize the marginal utility for a given set of $K$

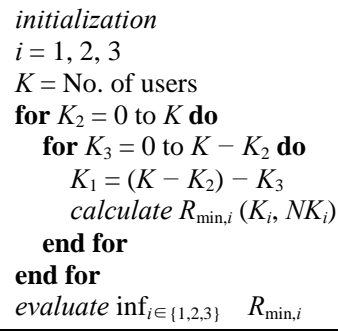

Algorithm 1. $R_{\min , i}$ for every value of $K_{1}, K_{2}$ and $K_{3}$ with $K=10$. contended users, i.e.,

$$
I_{\text {user }, k}^{(n)}=\arg \max _{k \in \mathcal{K}^{t}} \Lambda_{k}^{(n)}
$$

Three steps (user-RC) assignment is given below: \

Step I: Find the (user, RC) pair that gives highest marginal utility value

$$
\left[k^{*}, n^{*}\right]=\operatorname{argmax}_{k \in \mathcal{K}^{t}, n \in \mathcal{L}^{t}} \Lambda_{k, n}
$$

Step II: Allocate RC $n^{*}$ to user $k^{*}$

$$
\hat{I}_{\text {user }, k}^{(n)}=\left\{I_{\text {user }, k}^{(n)} \cup\left\{n^{*}\right\} \mid n^{*} \notin I_{\text {user }, k}^{(n)}\right\} \quad \forall k \in \mathcal{K}^{t}
$$

Step III: Delete the allocated RC from the available $\mathrm{RC}$ set $\mathcal{L}^{t}$

$$
\mathcal{L}^{t}=\mathcal{L}^{t}-I_{\mathrm{RC}, n}
$$

Repeat the steps I, II, and III, until all RCs are allocated.

\subsection{Complexity Analysis}

The proposed algorithm first determines the number of RCs and the number of RBs per RC for each group, then within the group it performs a linear search on the users and RCs in order to find the user-RC pair that maximizes the utility function. For proposed algorithm the result of $[8,(12)]$ modifies to the following:

$$
\begin{aligned}
\text { complexity } & \approx \mathcal{O}\left(\sum_{i=1}^{v} N_{\mathrm{RC}, K_{i}}^{2} K_{i}\right) \\
& \approx \mathcal{O}\left(\sum_{i=1}^{v}\left(\frac{N_{K_{i}}-r_{i}}{q_{i}}\right)^{2} K_{i}\right)
\end{aligned}
$$

where $q_{i}$ and $r_{i}$ are quotient and remainder from $N_{K_{i}} / K_{i}$, respectively, and $N_{\mathrm{RC}, K_{i}}$ is the number of RCs allocated to $K_{i}$ group. From the elementary calculus,

$$
\left\{a^{2}+b^{2}+c^{2}+\cdots\right\}<\{a+b+c+\cdots\}^{2}
$$

therefore, our proposed algorithm provides a gain of at least

$$
2\left(\frac{N_{K_{1}}}{q_{1}} \frac{N_{K_{2}}}{q_{2}}+\frac{N_{K_{2}}}{q_{2}} \frac{N_{K_{3}}}{q_{3}}+\frac{N_{K_{1}}}{q_{1}} \frac{N_{K_{3}}}{q_{3}}\right) .
$$

(when $r_{i}=0$ ) over the algorithm presented in [8].

\section{Performance Evaluation}

In this section, we provide computer simulations using MATLAB. Sum-throughput and individual user throughput have been compared with Round Robin (RR) and logarithmic utility based proportional fairness (PF) algorithm [12]. 


\subsection{Simulation Model}

The simulation model consists of a single cell with a eNB equipped with an omnidirectional antenna. The throughput is averaged over 1000 TTIs, with the duration of a TTI being $0.5 \mathrm{msec}$. The total bandwidth considered is $B=5 \mathrm{MHz}$, subdivided into $25 \mathrm{RBs}$ of 12 subcarriers each. 7 OFDM symbols in 1 TTI including the reference signal (RS). We consider a target BER of $10^{-3}$. The maximum mobile transmit power is considered to be 220 $\mathrm{mW}$. All mobiles are assumed to transmit at the maximum power, and the power is subdivided equally among all subcarriers allocated to the mobile.

\subsection{Simulation Results}

In Figure 4, we have $K=K_{1}+K_{2}+K_{3}=10$, i.e., the total number of users are 10. It has been observed that few users get the 16QAM constellation size due to narrow SNR band for this group, as shown in Figure 2. Therefore, we will be more interested in $K_{2}=0,1, \cdots, 5$ values. Solid line in first two sub-graphs in Figure 4 shows that when $K_{2}=0$ and $K_{3}=0$ all the RBs are allocated to users in group $K_{1}$ which results in more than 0.5 Mbps throughput to each user in this group, but with $K_{3}=1$ our algorithm allocates the RBs to users in this group with grouped share that is inversely proportional to the constellation size, which means the users with lower constellation size will get more RBs. Due to this reason, one user $K_{3}=1$ gets approximately $1 \mathrm{Mbps}$ and remaining 9 users of $K_{1}$ get increase in their throughput despite their poor channel condition. If majority of the users belong to group $K_{1}$, e.g., $K_{1}=9$ then almost all the RBs are assigned to this group, which usually requires more RBs than any other group to maintain the same throughput. This fact manifests from the lower throughput with larger values of $K_{1}$ and lower values of $K_{3}$. When $K_{2}=1$, the variation in $R_{\min }$ for users in $K_{1}$ and $K_{3}$ groups is shown with cross (x) marker dotted lines. In this case, $K_{3}$ graph starts from 9 users and $K_{1}$ graph stars with 0 . In $K_{3}$ graph, at $K_{2}=9$ the value of $R_{\min }$ is higher than the value with $K_{2}=0$, because now $K_{3}$ is competing with $K_{2}$, which requires less number of RBs to maintain its throughput as compared to resource hungriest group $K_{1}$. $R_{\min }$ for $K_{2}$ group is shown in bottom graph, where the value of $R_{\min }$ remains in the range of 2.5 to 1.5 Mbps due to proposed fairness algorithm.

In Figure 5 individual user throughput has been shown with distance from the eNB. Our proposed algorithm provides an increased fairness index ${ }^{1}$ of 0.3735 as

${ }^{1}$ Fairness = average user data rate at the cell boundary/average user data ratelcite\{Proportional Fair Scheduling of Uplink.\}
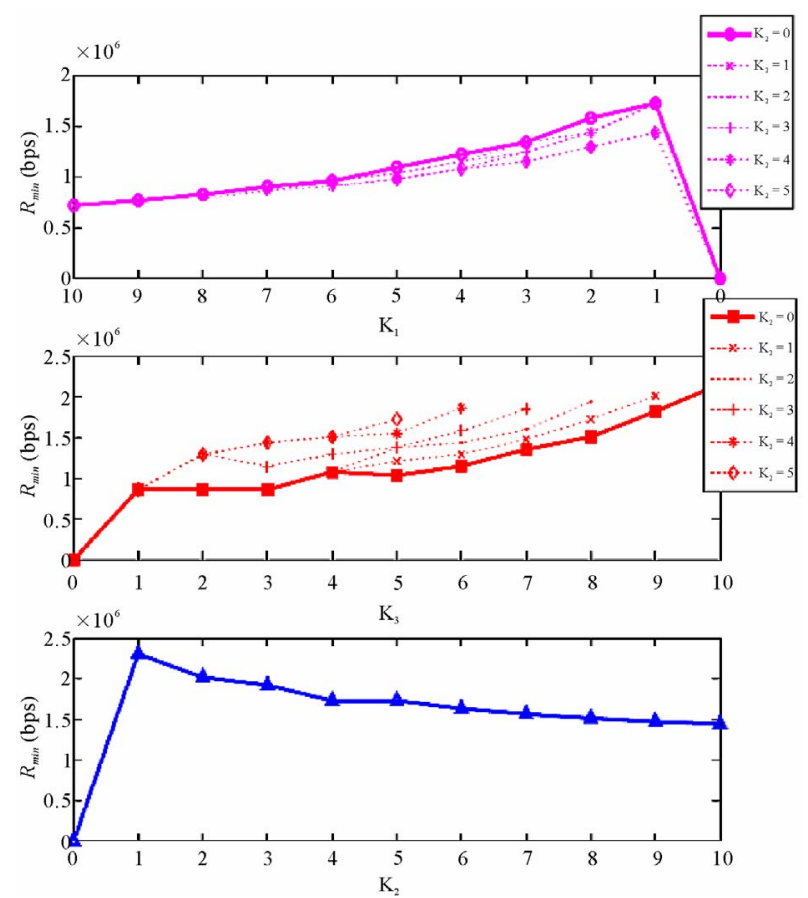

Figure 4. Group wise $R_{\min }$ for various values of $K_{1}, K_{2} . K_{3}$.

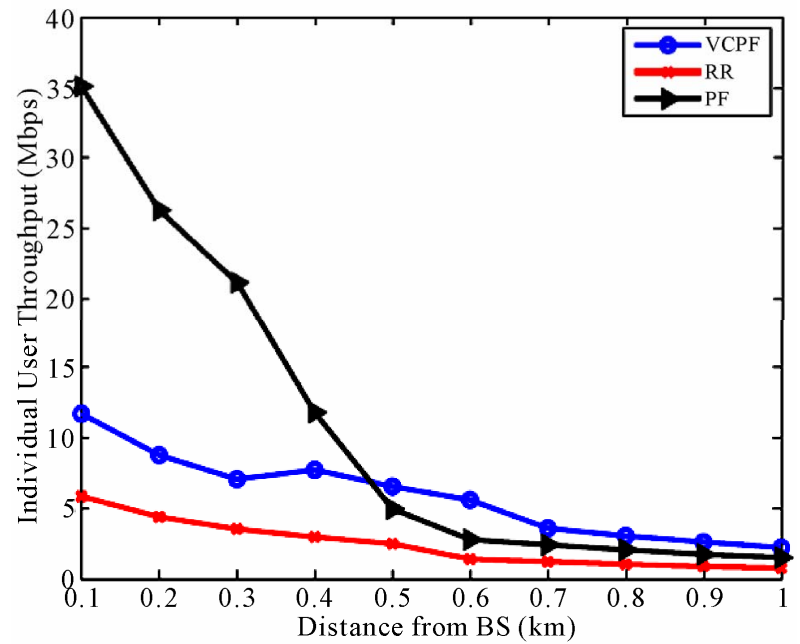

Figure 5. Throughput achieved by each user as a function of distance from the eNB.

compared to 0.1351 value for PF. The PF algorithm provides higher throughput to the users near to eNB, but VCPF outperforms PF after $0.5 \mathrm{Km}$ and provides higher throughput to the distant users even with poor channel condition. This can be achieved by proportional group based RB allocation in VCPF. Round Robin (RR) scheme has highest fairness factor but poorest throughput performance.

Figure 6 shows the sum-throughput of VCPF, PF, and RR schedulers. With one user in a coverage area of eNB, performance of all schemes is the same because they 


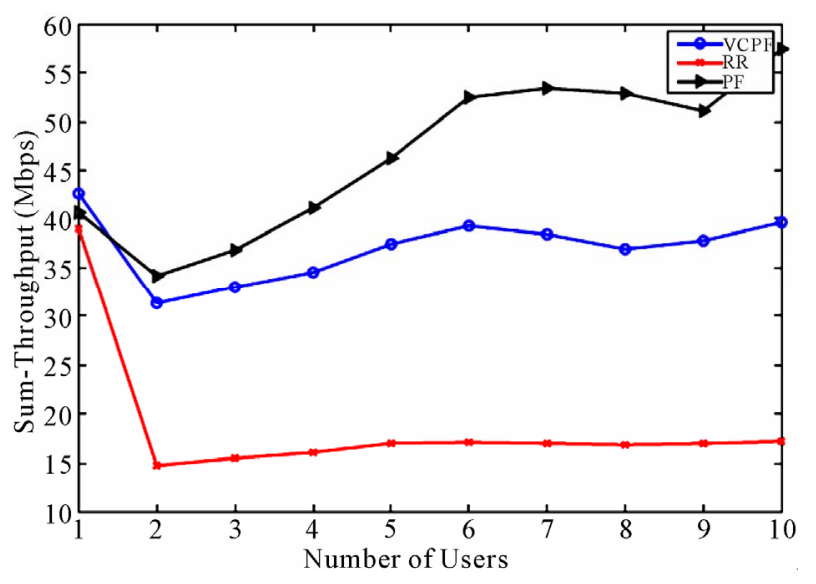

Figure 6. Sum-throughput of VCPF, PF, and RR schemes with $5 \mathrm{MHz}$.

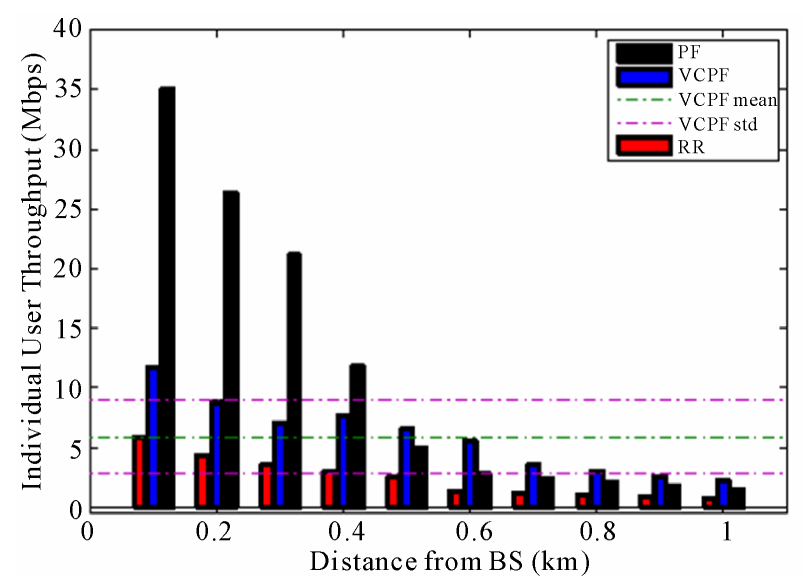

Figure 7. VCPF fairness-throughput compromise at various distances from eNB.

allocate all resources to that user, but as the number of users increases, RR allocates RCs to users in cyclic sequential manner, $\mathrm{PF}$ allocates the RBs to the user who has higher $\ln (R)$ and VCPF uses proportional group based RB reservation and then $\ln (R)$ based RC allocation. Since PF provides a higher throughput to users near to the eNB (high channel gain) and VCPF gives high priority to the distant users, therefore PF has high ratesum capacity. Increasing the number of users with user's placement proportional to coverage area results in more users in outer ring of coverage area and fewer users in the area close to the eNB. In this type of increase in users $\mathrm{PF}$ shows increasing trend with the number users, because it start allocating more resources to closest users with good channel conditions.

From Figure 7, it can be seen that VCPF provides average throughput of $5.888 \mathrm{Mbps}$ with standard deviation (STD) of 3.087, while PF and RR give average throughput of 10.97Mbps with STD of 12.26 and 2.439 Mbps with STD of 1.728, respectively. Hence our propose technique provides a compromise between throughput and fairness in comparison to the two extremes i.e., RR and PF.

It's worth noting here that VCPF is more attractive for cellular operators when QoS guarantees are critical compared to enhancing system capacity. It's also worth noting that VCPF can provide an efficient performance knob to the cellular operator to control the trade-off between fairness and system capacity by controlling the number of RBs (i.e. $N_{K_{i}}$ in (10)) assigned to each user group.

\section{Conclusions}

This paper presents a proportional group based radio resource allocation scheme. It uses MAC layer MCS information for the virtual cluster formation. Each cluster or group has been assigned a number of RBs. Since LTE SC-FDMA requires contiguous RB allocation, consecutive RBs form the resource chunks. User-RC are selected through proportional fairness algorithms within each group. Proposed algorithm can take part in the service level agreement (SLA) management between users and cellular operator. Simulation results show that this scheme can render the fairness much better than existing schemes. It has been shown that proposed algorithm has a complexity in the order of

$\ln (R) \mathcal{O}\left(\sum_{i=1}^{v}\left(\frac{N_{K_{i}}-r_{i}}{q_{i}}\right)^{2} K_{i}\right)$ i.e., the algorithms has linear complexity in the number of users and quadratic complexity in the RCs, and could be easily implemented in real time.

\section{References}

[1] S. Parkvall, E. Dahlman, A. Furuskar, Y. Jading, M. Olsson, S. Wänstedt and K. Zangi, "LTE-Advanced Evolving LTE towards IMT-Advanced," Proceedings of the IEEE Vehicular Technology Conference, Calgary, 21-24 September 2008, pp. 1-5.

[2] 3GPP TR 36.913 V.9.0.0, "Requirements for Further Advancements for Evolved Universal Terrestrial Radio Access (E-UTRA) (LTE-Advanced),” December 2009.

[3] 3GPP TR 36.814 V.9.0.0, "Further Advancements for E-UTRA Physical Layer Aspects,” Release 9, March 2010.

[4] J. S. Lim, H. G. Myung, K. J. Oh and D. J. Goodman, "Channel Dependent Scheduling of Uplink Single Carrier FDMA Systems,” 2006 IEEE 64th Vehicular Technology Conference, Montreal, 25-28 September 2006, pp. 1-5.

[5] L. Ruiz de Temino, G. Berardinelli, S. Frattasi and P. Mogensen, "Channel-Aware Scheduling Algorithms for SCFDMA in LTE Uplink," IEEE 19th International Symposium on Personal, Indoor and Mobile Radio Communi- 
cations, Cannes, 15-18 September 2008, pp. 1-6.

[6] C. Y. Ng and C. W. Sung, "Low Complexity Subcarrier and Power Allocation for Utility Maximization in Uplink OFDMA Systems," IEEE Transactions on Wireless Communications, Vol. 7, No. 5, 2008, pp. 1667-1675. doi:10.1109/TWC.2008.060723.

[7] F. D. Calabrese, P. H. Michaelsen, C. Rosa, M. Anas, C. U. Castellanos, D. L. Villa, K. I. Pedersen, P. E. Mogensen, "Search-Tree Based Uplink Channel Aware Packet Scheduling for UTRAN LTE," IEEE Vehicular Technology Conference, Singapore, 11-14 May 2008, pp. 1949-1953.

[8] E. Yaacoub and Z. Dawy, "A Game Theoretical Formulation for Proportional Fairness in LTE Uplink Scheduling," IEEE Wireless Communications and Networking Conference, Budapest, 5-8 April 2009, pp. 1-5.

[9] J. Huang, V. G. Subramanian, R. Agrawal and R. Berry,
"Joint Scheduling and Resource Allocation in Uplink OFDM Systems for Broadband Wireless Access Networks," IEEE Journal on Selected Areas in Communications, Vol. 27, No. 2, 2009, pp. 226-234. doi:10.1109/JSAC.2009.090213

[10] H. G. Myung, J. S. Lim and D. J. Goodman, "Single Carrier FDMA for Uplink Wireless Transmission,” IEEE Vehicular Technology Magazine, Vol. 1, No. 3, 2006, pp. 30-38. doi:10.1109/MVT.2006.307304

[11] A. Goldsmith, "Wireless Communications,” Oxford University Press, Oxford, 2005.

[12] J. S. Lim, H. G. Myung, K. J. Oh and D. J. Goodman, "Proportional Fair Scheduling of Uplink Single-Carrier FDMA Systems," IEEE 17th International Symposium on Personal, Indoor and Mobile Radio Communications, Helsinki, 11-14 September 2006, pp. 1-6. 